\title{
Modification of Free Volume due to Rigidness of Polymeric Matrix and Effects on Gas Transport Properties
}

\author{
R. Checchetto ${ }^{a, *}$ And R.S. Brusa ${ }^{a, b}$ \\ ${ }^{a}$ Department of Physics, University of Trento, via Sommarive 14, I-38123 Povo, Trento, Italy \\ ${ }^{b}$ TIFPA-INFN, via Sommarive 14, I-38123 Povo, Trento, Italy
}

\begin{abstract}
Gas barrier properties of polymeric membranes with different rigidness of their matrix where studied by gas phase permeation measurements. Gas transport results in membranes made of epoxy resin with different cross linking densities and epoxy resin with dispersed few layer graphene fillers were discussed and compared in the framework of the free volume theory of diffusion. Transport in cellulose membranes was found to occur in the diffusion configurational regime. The physical description of the transport properties was based on positron annihilation lifetime spectroscopy measurements which allowed to evaluate experimentally the fractional free volume in epoxy resin membranes and the size of rigid elongated cavities in cellulose thin films.
\end{abstract}

DOI: 10.12693/APhysPolA.132.1490

PACS/topics: 82.35.Lr, 61.25.hp, 36.10.Dr, 78.70.Bj

\section{Introduction}

Gas transport through polymeric materials is an important subject in different technological field as the development of selective membranes for gas separation and gas barrier coating for food packaging [1]. In particular, for food packaging, innovative materials produced using green technologies are required [2].

Mass transport processes in polymers are controlled by their free volume structure [3]. The thermal fluctuation of the polymer chain segments forms holes with sub-nanometric dimensions which can accommodate the permeant molecules. This non-static structure is called "free volume" of the polymer. The gas transport takes places by jumping of the permeant molecules between adjacent holes, and, if the fractional free volume is known, transport parameters such as diffusivity and permeability can be modelled in the frame of the free volume theory of diffusion [3]. In some organic polymers the matrix is rigid and the transport of permeant molecules is through connected cavities. Transport must be modelled by considering the different cavity shapes that depend on the type of material [4].

In this report we present a comparative discussion of transport properties in: (i) epoxy resin, a rubbery polymer, in which rigidification of the matrix was obtained by changing their cross-linking density $X_{c}$, (ii) dispersing graphene nanoplatelets in the epoxy resin, and (iii) an organic polymer, cellulose, in which rigid sub-nanocavities are present.

Transport properties were studied by gas phase permeation measurements: a detailed description of the experimental procedure and data analysis will be reported.

\footnotetext{
* corresponding author; e-mail: riccardo.checchetto@unitn.it
}

The open volume structure of the different polymer was characterized by PALS technique. Diffusion and permeation data have been modelled using the concentration, the size of hole (or cavities) and the free volume fraction as evaluated by PALS measurements.

\section{Experimental}

\subsection{Samples}

Three groups of samples were synthesized:

1. Epoxy resin membranes with four different cross linking density, were prepared using neopentyl glycol diglycidyl ether (NPGDE) (Sigma-Aldrich S.r.l., Italy) and a poly oxypropylene diamine as a cross-linking agent: Jeffamine D-230, D-400, ED600, ED-900 (Huntsman Chemical Co). Details on the preparation procedure and on the characterization of the sample by the Fourier transform infrared (FTIR), differential scanning calorimetry (DSC) and scanning electron microscopy (SEM) techniques can be found in Ref. [5]. The samples had the following cross linking density $X_{c}$ [site $/ \mathrm{nm}^{3}$ ]: 0.9 (NPGDE900), 1.15 (NPGDE600), 1.42 (NPGD400), and 1.67 (NPGDE230).

2. Nanocomposite membranes were synthesized by dispersing in the NPGDE-ED900 epoxy resin few layer graphene (FLG) nanoplatelets. Four samples with filler content equivalent to $0.6,2.8,4.3$ and 5.7 vol.\% were prepared. Details on the sample preparation procedure and on the characterization of fillers and nanocomposites by X-ray diffraction (XRD) and SEM techniques can be found in Ref. [6].

3. Carboxylated cellulose nanofibrils (TO-CNF) layers $(2.6,3.9,5.2,6.5 \mu \mathrm{m}$ thick) were deposited on $23 \mu \mathrm{m}$ thick polylactic acid (PLA) films. Details on preparation of the bilayer membrane 
TO-CNF/PLA and its characterization by atomic force microscopy (AFM) and SEM are reported in Ref. [7]. The TO-CNF are found to have an average width of $4 \mathrm{~nm}$ and length in the $50-300 \mathrm{~nm}$ range; nanofibrils are oriented with their long axis parallel to the PLA surface. The TO-CNF coating results homogeneous and with a flat surface.

\subsection{Positron annihilation lifetime spectroscopy}

Positron annihilation lifetime spectroscopy (PALS) measurements on epoxy resin membrane $12 \mathrm{~mm} \times 10 \mathrm{~mm}$ $\times 2 \mathrm{~mm}$, were carried out using a fast-fast coincidence lifetime setup (260 ps time resolution). The ${ }^{22} \mathrm{Na}$ source, deposited between two $7 \mu \mathrm{m}$ Kapton foils, was sandwiched between two equal samples. PALS spectra $\left(3 \times 10^{6}\right.$ annihilation events) were acquired by keeping the samples in vacuum $\left(10^{-5} \mathrm{~Pa}\right)$ at temperatures ranging between 183 and $323 \mathrm{~K}$ (uncertainty $\pm 1 \mathrm{~K}$ ) [8].

Depth-profiled PALS measurements on thin TO-CNF, as a function of energy (1-12 keV) and temperature (290-330 K), were carried out with pulsed low energy positron system (PLEPS) apparatus [9] at the high intensity positron source NEPOMUC (NEutron induced POsitron source MUniCh), by acquiring spectra containing $4 \times 10^{6}$ counts, and with a time resolution (pulsing and detector) of 230-240 ps.

\subsection{Gas transport measurements}

The gas transport properties are studied using samples shaped in form of thin disc with diameter $d \approx 1[\mathrm{~cm}]$ and thickness $h \approx 10$ to $100 \mu \mathrm{m}$ by gas phase permeation technique. At fixed temperature $T$ of the gas-sample system, at time $t=0$ (trigger time of the experiment) one side of the membrane, called high pressure side (HPS), is exposed to the permeant gas at fixed pressure $P_{\text {HPS }}$ while the other side, called low pressure side (LPS), faces an evacuated UHV analysis chamber (background pressure $P_{\text {LPS }}^{\text {back }}$ in the $10^{-6} \mathrm{~Pa}$ order). Gas molecules are absorbed in the HPS layers of the membrane, diffuse through the membrane layers, reach the LPS layers and desorb in the UHV chamber where the pressure $P_{\text {LPS }}(t)$ increases as a function of time according to the following relation [10]:

$$
Q(t)=V \frac{\mathrm{d} P_{\mathrm{LPS}}(t)}{\mathrm{d} t}+S_{p} P_{\mathrm{LPS}}(t),
$$

where $Q(t)$ is the inlet flow of permeant molecules, $V$ the volume of the UHV chamber and $S_{p}$ - the pumping speed of the vacuum system. Thermal equilibrium conditions between permeated molecules and analysis chamber are immediately set by collision processes with the chamber walls: experimental transport data can be thus reported in terms of molar permeation flux, $j_{\text {perm }}(t)$, through the relation:

$$
j_{\text {perm }}(t)=Q(t) /\left(A R T_{\text {chamb }}\right),
$$

where $R$ is the universal gas constant, $T_{\text {chamb }}$ is the temperature of the chamber walls, which is generally near room temperature, and $A$ is the membrane surface area. A background flow, $Q_{b a c k}$, is always present in the
UHV chamber due to outgassing processes of the chamber walls. We have used two experimental procedures to measure $j_{\text {perm }}(t)$ :

1. Constant volume $(\mathrm{CV})$ : at the trigger time $t=0$ the pumping system is excluded by closing a valve $\left(S_{p}=0\right)$ and the pressure signal $P_{\text {LPS }}(t)$ is recorded as function of time. In Sect. 4, permeation curves measured in CV mode are reported (Fig. 1, upper part). According to Eq. (1) and Eq. (2), the permeation flux $j_{\text {perm }}(t)$ is proportional to the rate of pressure increase $\frac{\mathrm{d} P_{\mathrm{LPS}}(t)}{\mathrm{d} t}$.

2. Constant pumping $(\mathrm{CP})$ : when the pumping speed $S_{p}$ satisfies the condition $S_{p} / V \gg \frac{1}{P_{\mathrm{LPS}}} \frac{\mathrm{d} P_{\mathrm{LPS}}(t)}{\mathrm{d} t}$, then the $P_{\text {LPS }}(t)$ signal as a function of time is proportional to the permeation flux, providing a direct measure of it. In Sect. 4, a permeation curve measured in CP mode is reported (Fig. 5).

The CV procedure provides accurate measurements when the pressure increase in the analysis chamber due to the permeation process $\frac{\mathrm{d} P_{\mathrm{LPS}}(t)}{\mathrm{d} t}$ is much larger than the pressure increase due to the background flow $\frac{\mathrm{d} P_{\mathrm{LPS}}^{b a c k}(t)}{\mathrm{d} t}$, which is evaluated setting $P_{\mathrm{HPS}}=0$. The signal-tonoise ratio $S / N=\frac{\mathrm{d} P_{\mathrm{LPS}}(t)}{\mathrm{d} t} / \frac{\mathrm{d} P_{\mathrm{LPS}}^{\text {back }}(t)}{\mathrm{d} t}$ is large enough to ensure reliable measurements with membrane samples made of highly gas permeable materials. In this procedure, $P_{\text {LPS }}(t)$ is usually measured by using a total pressure gauge (spinning rotor gauge).

The CP procedure allows detailed and accurate measurements also with low permeability materials. The continuous pumping of the analysis chamber ensures UHV conditions during the experimental run and permits the use of quadrupole mass spectroscopy (QMS) techniques to measure the partial pressure signal of the permeating gas $P_{\text {LPS }}(t)$, resolving it from the partial pressure of those gases forming the background pressures $P_{\mathrm{LPS}}^{\text {back }}$.

In the $\mathrm{CP}$ procedure, the signal-to-noise ratio is given by $S / N=P_{\mathrm{LPS}} / \delta P_{\mathrm{LPS}}^{\text {back }}$ where $\delta P_{\mathrm{LPS}}^{\text {back }} \ll P_{\mathrm{LPS}}$ are the fluctuations of the background partial pressure $P_{\mathrm{LPS}}^{\text {back }}$ of the permeating gas while the quantity $\delta J_{\text {perm }}=$ $S_{p} \delta P_{\mathrm{LPS}}^{\text {back }} /\left(A R T_{\text {chamb }}\right)$ provides an estimate of the system detection limit.

We used the CV procedure in the case of epoxy resin membrane while $\mathrm{CP}$ procedure was mandatory for the measurement with TO-CNF/PLA membranes.

\section{Data analysis}

$$
\text { 3.1. PALS }
$$

PALS spectra were analyzed using PATFIT-88 routine analysis [11]. All spectra were well fitted with three components. In the following we consider only the intensity and lifetime of the long component $\left(\tau_{3}, I_{3}\right)$ pertinent to the pick-off annihilation of ortho-positronium (o-Ps) which is related to concentration and size of free volumes. The hole free volume dimension $\nu_{h}$ in epoxy-resin and the 
size $d$ of nanochannels in TO-CNF were obtained by the quantum relation between pick-off lifetime $\tau_{3}$ and void dimension $[12,13]$. A typical measure of $\tau_{3}, I_{3}$ as a function of $T$ is shown in Fig. 2. The important parameters that are evaluated from PALS spectra measurements and allow to describe the permeation processes on the basis of the free volume structure of the polymer are (see Fig. 2 and Ref. [8]):

(a) The variation of $I_{3}$ that can be related to the concentration of free holes or pores [14].

(b) The values of $\nu_{h}$ and $d$, which can be compared with the kinetic diameter (in $\AA$ ) of the penetrant gases $\left(\sigma_{\mathrm{He}}=2.6, \sigma_{\mathrm{D}}=2.89, \sigma_{\mathrm{N}_{2}}=3.64, \sigma_{\mathrm{CO}_{2}}=3.3, \sigma_{\mathrm{O}_{2}}=\right.$ $3.46)$ to evaluate the accessibility of atom/molecules to the free volumes.

(c) The fractional free volume $f_{h}(T)$ :

$$
f_{h}(T)=\frac{I_{3}(T)}{I_{3}\left(T_{g}\right)}\left[\frac{\alpha_{V}}{\alpha_{h r}}+\alpha_{V}\left(T-T_{g}\right)\right]
$$

that can be calculated $[8,15,16]$ measuring the thermal expansion coefficient $\alpha_{V}$, and obtaining (i) the $I_{3}(T)$, $I_{3}\left(T_{g}\right)$ values and, by the direct analysis of $v_{h}$ vs. $T$ curves, (ii) the thermal expansion coefficient of the hole free volume $\alpha_{h}=\frac{1}{\nu\left(T_{g}\right)}\left(\frac{\mathrm{d} \nu_{h}}{\mathrm{~d} T}\right)\left(T>T_{g}\right)$, (iii) the $T_{g}$, $\nu_{h}\left(T_{g}\right)$ values. The $T_{g}$ and $\nu_{h}\left(T_{g}\right)$ values are found by the intersection of the two linear fits to the linear variation of the hole free volume $v_{h}$ in the glassy phase $\left(T<T_{g}\right)$ and in the rubbery phase $\left(T>T_{g}\right)$ while $\frac{\mathrm{d} \nu_{h}}{\mathrm{~d} T}$ is given by the slope of the linear fit for $T>T_{g}$.

The $f_{h}(T)$ values are experimental input values for application of free volume theory to calculate permeability, diffusivity and permeability.

\subsection{Gas transport}

In our experiment: (1) the membrane thickness, along which penetrant migration occurs, is much shorter than the disc diameter, and the edge effect can be neglected; (2) gas transport was found to obey the solution-diffusion mechanism $[5,7]$.

According to the first condition the experimental results can be analyzed in a one-dimensional geometry and the transport problem modelled assuming [1] that penetrant diffusion obeys the Fick law $\frac{\mathrm{d} c(x, t)}{\mathrm{d} t}=D \frac{\mathrm{d}^{2} c(x, t)}{\mathrm{d} x^{2}}$ where $D$ is the diffusion constant and $c(x, t)$ is the penetrant concentration in the membrane layers at depth $x$ below the membrane surface and at time $t$ after the trigger time. The second condition indicates that penetrant solution obeys to the Henry law: consequently $c(x, t)$ at the membrane boundaries in $x=0$ and $x=h$, is given by $c(x=0, t)=\Pi P_{\mathrm{HPS}}$ and $c(x=h, t)=\Pi P_{\mathrm{LPS}}$, where $\Pi$ is the penetrant solubility.

The Fick equation (i), with boundary condition given in (ii), can be analytically solved. When the condition $P_{\text {HPS }} \gg P_{\text {LPS }}$ holds, as it generally occurs in gas phase permeation experiments, the molar permeation flux is given by the relation [17]:

$$
j_{\text {perm }}(t)=j_{\text {stc }}\left[1+2 \sum_{n=1}^{\infty}(-1)^{n} \mathrm{e}^{-\frac{D n^{2} \pi^{2} t}{h^{2}}}\right],
$$

where $j_{\text {stc }}=\frac{1}{h} D \Pi P_{\mathrm{HPS}}$ is the permeation flux in stationary transport conditions (stc), that is when $t \rightarrow \infty$.

The previous Eq. (4) is directly used to fit the experimental transport data and evaluate the $D$ and $\Pi$ parameters when the gas transport process is studied using the $\mathrm{CP}$ procedure.

When gas transport is studied using the CV procedure a different approach is used. As long as permeated molecules in the analysis chamber form a rarefied gas, pressure changes with time according to the relation

$$
P_{\text {LPS }}(t)=\frac{R T_{\text {chamb }}}{V} A M(t),
$$

where $M(t)$ is the total number of permeated gas moles per unit surface area of the membrane sample at time $t$ which is given by

$$
\begin{gathered}
M(t)=\int_{0}^{t} j_{\text {perm }}(t) \mathrm{d} t=j_{\text {stc }}\left(t-\frac{h^{2}}{6 D}\right) \\
-2 h \frac{\Pi P_{\mathrm{HPS}}}{\pi^{2}} \sum_{n=1}^{\infty} \frac{(-1)^{n}}{n^{2}} \mathrm{e}^{-\frac{D n^{2} \pi^{2} t}{h^{2}}} .
\end{gathered}
$$

In stc conditions the following approximation for $M(t)$ holds: $M(t)=\frac{1}{h} D \Pi P_{\mathrm{HPS}}\left(t-\frac{h^{2}}{6 D}\right)$. Consequently the intercept, with the time axis, of the straight line fitting the $P_{\text {LPS }}(t)$ data provides the so-called time lag value $\tau_{L}=\frac{h^{2}}{6 D}$, and permits to evaluate the diffusion coefficient $D$. The membrane permability $\Phi=\Pi D$, can be obtained by the slope of the $P_{\text {LPS }}(t)$ signal in stc (Fig. 1a).

\section{Results and discussion}

Permeation measurement in epoxy-resin with different cross linking density $X_{c}$ were carried out with $\mathrm{H}_{2}, \mathrm{~N}_{2}$ and $\mathrm{CO}_{2}$ as penetrant molecules. Two typical experimental curves are reported in Fig. 1, upper part, for the NPGED900 sample presenting the lowest $X_{c}$ : the pressure $P_{\text {LPS }}$ rise as a function of time is shown for two different $P_{\text {HPS }}$ pressure when the membrane is exposed to $\mathrm{CO}_{2}$. The linear behavior at different $P_{\mathrm{HPS}}$ is an indication that the transport obeys the solution-diffusion mechanism. The mean permeation $\Phi$ and diffusion $D$ values have been obtained from the slope and the time lag (see Sect. 3.2) of at least three measurement at different $P_{\text {HPS }}$. In Fig. 1, lower part, the $\Phi$ and $D$ values obtained by changing the sample temperature are shown in an Arrhenius plot, which give the activation energy for permeation and diffusion. The activation energy for each gas, were found to increase by increasing the $X_{c}[5,8,16]$.

The pick-off $o$-Ps lifetime and intensity for the sample with the lowest (NPGED900) and highest (NPGD230) $X_{c}$, are shown in Fig. 2 as a function of the sample temperature. In the same figure, the hole free volume $v_{h}$ is also reported. Above $T_{g}$, the diameter of the free holes of volume $v_{h}$ are larger than $4 \AA$. Because this value is bigger than all kinetic diameter of the molecules, these holes 

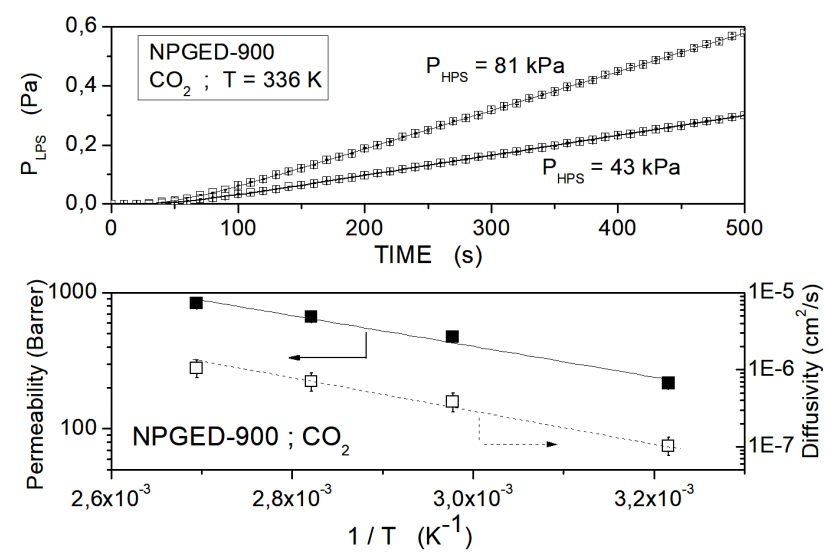

Fig. 1. Upper part: $P_{\text {LPS }}$ rise measured (CV procedure) with the NPGED900 sample exposed to $\mathrm{CO}_{2}$ at two different $P_{\text {HPS }}$ values. Line, curves from which $\Phi$ and $D$ values are evaluated. Lower part: Arrhenius plot for permeability $\Phi$ and diffusivity $D$ values for $\mathrm{CO}_{2}$.

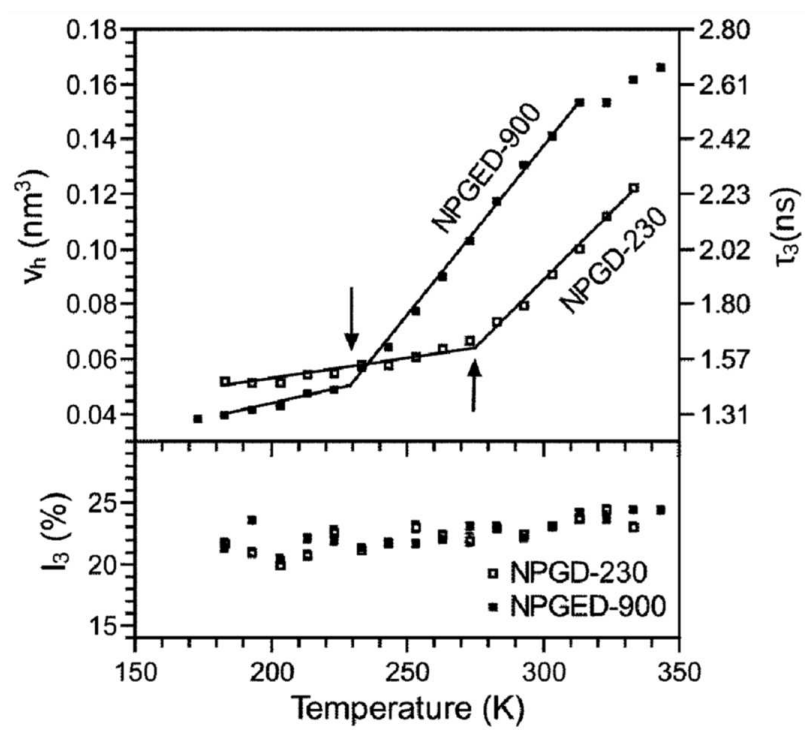

Fig. 2. Upper part: o-Ps lifetime values $\tau_{3}(T)$ and hole volumes $v_{h}(T)$ for the NPGED900 and NPGED230 samples. The intersection of the fitting lines of the rubbery and glassy phase determine the $T_{g}$. Lower part: Lifetime intensity $I_{3}(T)$.

can accommodate all the considered permeant molecules in their diffusion.

According to the diffusion free volume theory [3, 4], the experimental transport data can be modelled by the following relation that put in relation the gas permeation with the free volume fraction $f_{h}(T)$ as obtained by PALS data (see Sect. 3.1):

$$
\begin{aligned}
& \Phi(T)=D(T) \Pi(T)= \\
& \Phi_{0} \exp \left(\frac{-B_{d}}{f_{h}(T)}\right) \exp \left(\frac{-\Delta H_{S}}{R T}\right),
\end{aligned}
$$

where the first exponential, in which $B_{d}$ is a parameter that depend from the polymer-penetrant system, relates the diffusivity to $f_{h}(T)$, and the second exponential de- scribes the solubility dependence from $T$ and the sorption enthalpy $\Delta H_{S} \cong-\Delta H_{V}$, with $-\Delta H_{V}$ the vaporization enthalpy of the gas [16].

In Fig. 3 we show, as an example, a fit to the $\mathrm{CO}_{2}$ permeability data as a function of $1 / f_{h}$ for the samples with the highest and lower $X_{c}$. The only free parameter was $B_{d}$. For each gas, it was found to be constant changing the $X_{c}: 0.59,0.51,0,37$ for $\mathrm{CO}_{2}, \mathrm{~N}_{2}, H_{2}$, respectively. At parity of free volume fraction, the permeability was found to be highest for $\mathrm{CO}_{2}$ and lowest for $\mathrm{N}_{2}$ : for example for $1 / f_{h}=11, \Phi=225,80,17.5$ (barrer) in $\mathrm{CO}_{2}$, $\mathrm{H}_{2}, \mathrm{~N}_{2}$, respectively, while for each gas the permeability decreases with the increase of the $X_{c}$ (Fig. 3). It can be inferred that the enhanced rigidity introduced by the increase of $X_{c}$ limits the distribution of free holes because the flexibility of the polymer chain diminishes.

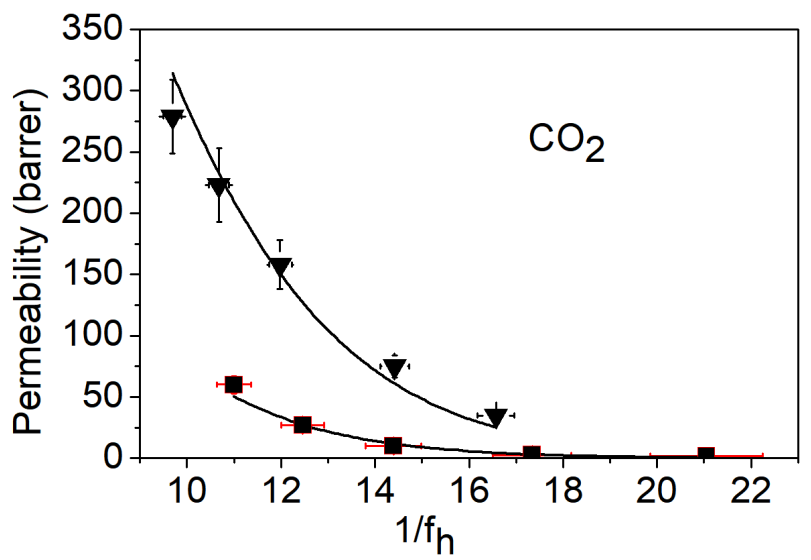

Fig. 3. Gas permeability of $\mathrm{CO}_{2}$ as a function of the inverse of the fractional free volume $f_{h}(T)$, for the NPGED900 (inverted triangles) and NPGED230 (squares) samples. The lines are fits with Eq. (6).

Successively a nanocomposite was studied adding FLG nanoplatelets, an impermeable filler, to NPGDE900 epoxy resin. This sample, with the highest permeability due to the lowest $X_{c}$, was chosen to have a better $\mathrm{S} / \mathrm{N}$ ratio in the transport measurements. A decrease in $\mathrm{CO}_{2}$ permeability was observed by increasing of the vol.\% of nanoplatelets (see Fig. 4), showing an improvement in the gas barrier property of the membrane.

On the other hand, PALS measurements pointed out that the $o$-Ps lifetime as a function of $T$, for $T>T_{g}$, did not change by varying the vol. $\%$ of filler, but they showed a decrease of the intensity $I_{3}$ from $\approx 22 \%$ in sample with 2.8 vol. $\%$ to $\approx 16 \%$ in sample with 5.7 vol. $\%$. These findings are a clear evidence that filler particles do not change the free volume structure of the nanocomposite but reduce the number of hole free volumes, and consequently the variation of the fractional free volume $f_{v} \propto \tau_{3} I_{3}$ is controlled by $I_{3}[8]$.

Different models were considered to explain the permeability data, among them: (i) the "tortuous path" model that attributes the reduction of permeability to the tortuous path, longer than the thickness of the membrane, 


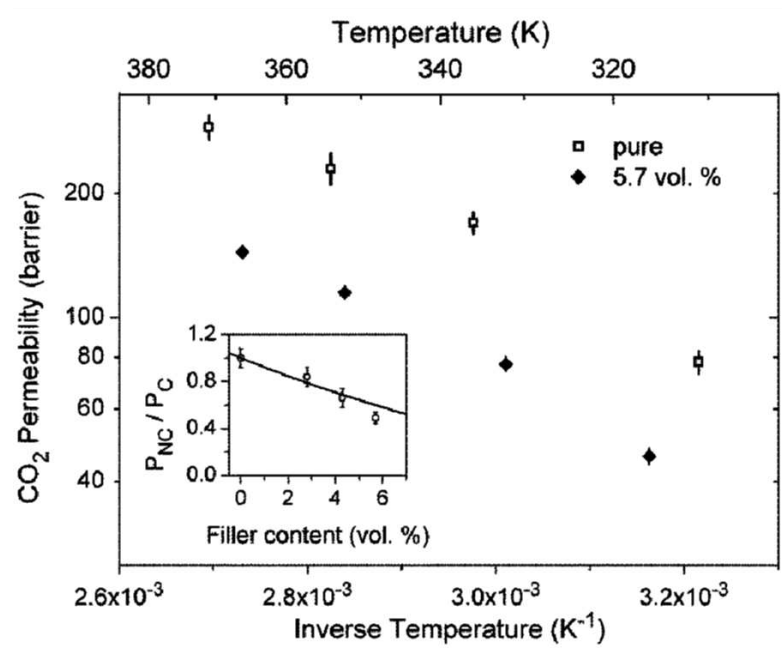

Fig. 4. $\mathrm{CO}_{2}$ permeability in pure and the loaded with 5.7 vol.\% FLG NPGED900 samples, as a function of $1 / T$. Inset: reduction of permeability as a function of filler content. The line is the theoretical evaluation with Eq. (8).

that molecules are forced to follow, (ii) the model considering free volumes at the interfacial layer between fillers and matrix. The only successful model, also compatible with the PALS results, was that considering a rigidified region of the polymer around the fillers. This rigidified region can be induced by the coupling between fillers and matrix.

The reduction of permeability in the nanocomposite (NC) respect to the epoxy without fillers can be expressed through geometrical parameters [8]:

$$
\frac{\Phi_{N C}}{\Phi}=\frac{1-\phi_{d}\left(1+\frac{2 l}{w}\right)}{1+\frac{1}{2} \phi_{d}\left(1+\frac{2 l}{w}\right)},
$$

where $l$ is the thickness of the rigidified region at each filler interface, $w$ - the thickness of the filler $(\approx 10 \mathrm{~nm})$, $\phi_{d}$ - the filler volume fraction (filler mean length $0.1 \mathrm{~mm}$ ). A thickness $l=20 \mathrm{~nm}$ was evaluated by the reduction of free volume as measured by PALS.

In the inset of Fig. 4 the experimental data of permeability reduction and the theoretical line obtained by Eq. (8) are shown. From these results we can expect that the inclusion of fillers in epoxy resin with the highest number of cross linking would reduce drastically the permeation.

The last system studied, the bilayer membranes with cellulose nanofibrils thin films, has shown strong barrier properties. $\mathrm{No}_{\mathrm{CO}_{2}}$ and $\mathrm{N}_{2}$ signal were detected in permeation measurements carried out in CP mode and lasting hours, meaning that the permeation flux was below the detection limit of $\approx 10^{-2} \mathrm{~mL} \mathrm{~m}^{-2}$ day $^{-1}$. In Fig. $5 \mathrm{a}$ permeation curve of TO-CNF/PLA membrane using ${ }^{2} \mathrm{H}_{2}$ as test gas is shown.

The average room temperature ${ }^{2} \mathrm{H}_{2}$ permeability and diffusivity were extremely low: $\Phi=0.10 \pm$ $0.03 \mathrm{~mL} \mu \mathrm{m} \mathrm{m}^{-2}$ day $^{-1} \mathrm{kPa}$ and $D=2.2 \pm 0.4 \times$ $10^{-10} \mathrm{~cm}^{2} \mathrm{~s}^{-1}$. To have a comparison, the diffusiv-

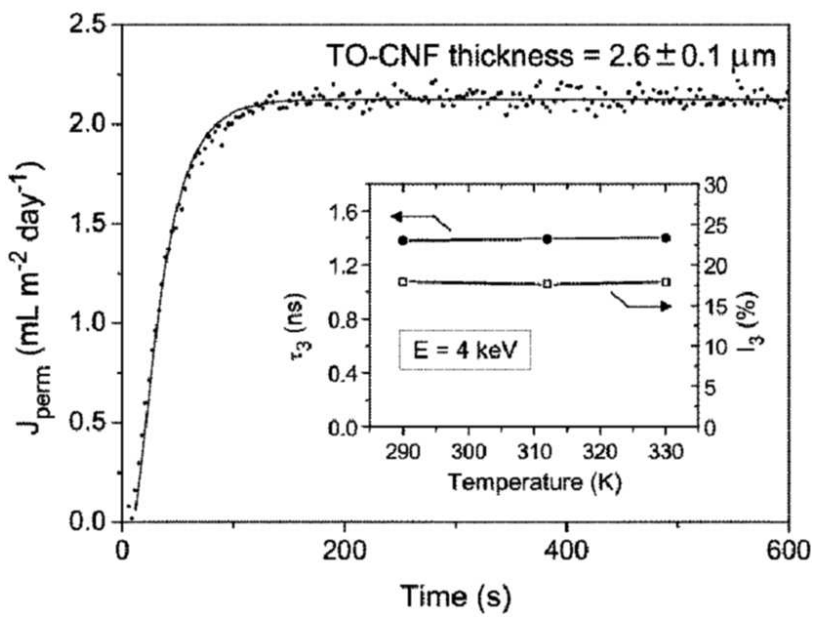

Fig. 5. Permeation curves, measured with the CP procedure, of TO-CNF/PLA membrane exposed to ${ }^{2} \mathrm{H}_{2}$. The thickness of TO-CNF is $2.6 \pm 0.1 \mu \mathrm{m}$. The line is the fit with Eq. (3). Inset: $o$-Ps lifetime $\tau_{3}(T)$ and intensity $I_{3}(T)$ values.

ity in PET is three order of magnitude higher: $D \approx$ $10^{-7} \mathrm{~cm}^{2} \mathrm{~s}^{-1}$. Permeation measurement as a function of $T$ allow to evaluate an energy of activation for diffusion $E_{D}=39 \pm 1 \mathrm{~kJ} \mathrm{~mol}^{-1}$.

In the inset of Fig. 5 we report the PALS measurement as a function of $T$ at a depth of $\approx 0.25 \mu \mathrm{m}\left(\mathrm{e}^{+}\right.$ implantation energy $4 \mathrm{keV}$ ). Both $\tau_{3}$ and $I_{3}$ are constant. Measurement as a function of depth also showed constant values [7].

Because the highly packed nanofibrils are gasimpermeable, the gas molecules can only diffuse through empty space among nanofibrils. These empty spaces can be inferred to be elongated cavities with the nanofibrils as walls. By $\tau_{3}$ value, modelling the cavity with a square section of size $d$ and length of the order of tens of $\mu \mathrm{m}$, $\gg d$, one can calculate [13] $d=0.31 \mathrm{~nm}$. If we compare this size with the kinetic diameter of the penetrant molecule we see that $\sigma_{\mathrm{He}}<\sigma_{\mathrm{D}}<d<\sigma_{\mathrm{CO}_{2}}<\sigma_{\mathrm{O}_{2}}<\sigma_{\mathrm{N}_{2}}$, suggesting that the barrier property of the TO-CNF can be attributed to a size-sieving effect. Moreover, $\sigma_{\mathrm{He}}, \sigma_{\mathrm{D}}>0.8 d$ and the diffusion occurs in "configurational regime": the molecule interact with the walls of the cavity, modelled as a corrugated potential with dips $>k_{\mathrm{B}} T$. Diffusion occur by thermal activated hopping between adjacent sites of the potential

$$
D=\frac{1}{2} v L \mathrm{e}^{\frac{-E_{D}}{k_{\mathrm{B}} T}}
$$

Giving a typical vibration of $v \approx 10^{-12} \mathrm{~s}^{-1}$, a distance $L \approx 1 \mathrm{~nm}$ between site can be estimated. This shows that the molecules make some thousands of jumps before moving in a second channel.

\section{Conclusions}

We have characterized the free volume structure of different polymeric systems, from synthetic to organic, by PALS technique. It was shown that the experimental 
evaluation of the hole free volume $v_{h}$, their concentration and the fractional free volume, permit to have a deeper insight on the nanoscopic mechanism governing the gas transport properties. The gas phase permeation data can be modelled using as input the PALS experimental finding, reducing the unknown free parameters. Very precise gas permeation measurements can be also performed in studying organic polymeric systems with high barrier properties, thanks the use of the quadrupole mass spectroscopy.

The methodology for analyzing the transport data (permeation and diffusion) by PALS and gas phase permeation experiments are expected to be very advantageous for studying selective membranes and, overall, new biocompatible organic polymer requested by the packaging industry.

\section{References}

[1] S. Matteucci, Y. Yampolskii, B.D. Freeman, I. Pinnau, in: Materials Science of Membranes for Gas and Vapor Separation, Eds. Y. Yampolskii, I. Pinnau, B.D. Freeman, Wiley, Chichester 2006, p. 1.

[2] V. Siracusa, P. Rocculi, S. Romani, M.D. Rosa, Trends Food Sci. Technol. 19, 634 (2008).

[3] J.L. Duda, J.M. Zielinsky, in: Diffusion in Polymers, Ed. P. Neogi, Marcel Dekker, New York 1996, p. 143.

[4] V.N. Burganos, in: Comprehensive Membrane Science and Engineering, Eds. E. Drioli, L. Giorno, Elsevier, Kidlington, UK 2010, p. 29.
[5] P.N. Patil, D. Roilo, R.S. Brusa, A. Miotello, R. Checchetto, Polymer 58, 130 (2015).

[6] D. Roilo, P.N. Patil, R.S. Brusa, A. Miotello, S. Aghion, R. Ferragut, R. Checchetto, Polymer 121, 17 (2017).

[7] D. Roilo, C.A. Maestri, M. Scarpa, P. Bettotti, W. Egger, T. Koschine, R.S. Brusa, R. Checchetto, J. Phys. Chem. C 121, 15437 (2017).

[8] P.N. Patil, D. Roilo, R.S. Brusa, A. Miotello, S. Aghion, R. Ferragut, R. Checchetto, Phys. Chem. Chem. Phys. 18, 317 (2016).

[9] P. Sperr, W. Egger, G. Kögel, G. Dollinger, C. Hugenschmidt, R. Repper, C. Piochacz, Appl. Surf. Sci. 255, 35 (2008).

[10] P.A. Redhead, J.P. Hobson, E.V. Kornelsen, The Physical Basis of Ultrahigh Vacuum, AIP, 1993.

[11] P. Kirkegaard, N.J. Pedersen, M. Eldrup, PATFIT88, Risoe Natl. Lab., Roskilde, Denmark 1989.

[12] M. Eldrup, D. Lightbody, J.N. Sherwood, Chem. Phys. 63, 51 (1981).

[13] B. Jasińska, A. Kozioł, T. Goworek, J. Radioanal. Nucl. Chem. 210, 617 (1996).

[14] P.E. Mallon, in: Positron and Positronium Chemistry, Eds. Y.C. Jean, P.E. Mallon, D.M. Schrader, World Sci., Singapore 2002, p. 253.

[15] H.A. Hristov, B. Bolan, A.F. Yee, L. Xie, D.W. Gidley, Macromolecules 29, 8507 (1996).

[16] D. Roilo, P.N. Patil, R.S. Brusa, A. Miotello, R. Checchetto, Polymer 113, 147 (2017).

[17] J. Crank, The Mathematics of Diffusion, Clarendon Press, 1979 